\title{
Linoleic acid: Is this the key that unlocks the quantum brain? Insights linking broken symmetries in molecular biology, mood disorders and personalistic emergentism
}

\author{
Massimo Cocchi ${ }^{1,2^{*}}$ (D) Chiara Minuto ${ }^{3}$, Lucio Tonello ${ }^{1}$, Fabio Gabrielli', Gustav Bernroider ${ }^{4}$, Jack A. Tuszynski ${ }^{5,6}$, \\ Francesco Cappello ${ }^{7,8}$ and Mark Rasenick ${ }^{9,10}$
}

\begin{abstract}
In this paper we present a mechanistic model that integrates subneuronal structures, namely ion channels, membrane fatty acids, lipid rafts, $G$ proteins and the cytoskeleton in a dynamic system that is finely tuned in a healthy brain. We also argue that subtle changes in the composition of the membrane's fatty acids may lead to down-stream effects causing dysregulation of the membrane, cytoskeleton and their interface. Such exquisite sensitivity to minor changes is known to occur in physical systems undergoing phase transitions, the simplest and most studied of them is the so-called Ising model, which exhibits a phase transition at a finite temperature between an ordered and disordered state in 2- or 3-dimensional space. We propose this model in the context of neuronal dynamics and further hypothesize that it may involve quantum degrees of freedom dependent upon variation in membrane domains associated with ion channels or microtubules. Finally, we provide a link between these physical characteristics of the dynamical mechanism to psychiatric disorders such as major depression and antidepressant action.
\end{abstract}

Keywords: Mood disorders, Linoleic acid, Ion channels, Cytoskeleton, Microtubule, Lipid raft, Depression, Antidepressants, Ising model, Quantum states

\section{Background}

The knowledge of the neuraxis (and, in particular, brain) anatomy and physiology is complicated by the fact that, still today, the heterogeneous population of neurons and glial cells is far from being properly characterized at a molecular level. Much attention, in the last decades, has been paid to genes and proteins, in terms of expression, subcellular localization and molecular pathways and trafficking, but many studies neglected to focus on another, ancestrally basic, component of the nervous cells, the lipids that not only form the barrier between the internal and external environment but also have a dramatic role in the homeostasis of the entire nervous environment.

\footnotetext{
*Correspondence: massimo.cocchi@unibo.it

1 "Paolo Sotgiu" Institute for Research in Quantitative \& Quantum

Psychiatry \& Cardiology, L.U.de.S. HEl, Malta, Switzerland

Full list of author information is available at the end of the article
}

Since 1929 when Burr and Burr demonstrated the characteristics of essentiality of linoleic acid for animal organisms, some studies have tried to correlate the intake of this fatty acid with health status and, in particular, have investigated the functioning of the brain. In reality the presence of a minimum concentration of linoleic acid in the brain, together with the demonstration of its negligible transformation into the longer-chain derivatives and the low significance of linoleic acid diet on fatty acid composition of the brain, have not allowed, in the past, relevant interpretive access to its possible functional complexity in the brain.

Several studies on the fatty acid composition of the brain of many animals have consistently recorded concentrations around $1 \%$ [1-4]. Cocchi et al. [5] have tried to explain such a reduced concentration of linoleic acid as a stabilization element of the membranes, recognizing that this property is at least partially due to its 
melting point $\left(-5^{\circ} \mathrm{C}\right)$ and its spatial dimension. Its melting point places it as an intermediate between palmitic acid $\left(+63{ }^{\circ} \mathrm{C}\right)$ and arachidonic acid $\left(-50^{\circ} \mathrm{C}\right)$, that is, the extremes of the most significant fatty acid profile of the brain, conferring and maintaining an appropriate level of mobility of the membrane, for example, as occurs in extreme environmental conditions with regard to temperature $[6,7]$.

We offer as the most likely explanation for the extremely low concentrations of linoleic acid, in the normal terrestrial habitat, a fortiori, the essential role such a fatty acid could play as an element that controls a symmetry breaking effect (representing a bifurcation point for the system at which two qualitatively different global behaviors can ensue) for cell membranes in the neurons and glia. This situation, involving extreme sensitivity to small, even infinitesimal changes in the control parameter values, determining the system's response in terms of its dynamical behavior, is well known in the physics of phase transitions. Near a critical state (also called a transition point) in systems undergoing phase transitions [8] a single physical control parameter (e.g. pressure, temperature, or relative concentrations of chemical species in a mixture), determines the nature of the equilibrium state of the physical system under consideration. A classic example involves a magnetic system composed of tiny magnets represented by spins arranged geometrically in a regular array. In principle they can be oriented in arbitrary directions but they also interact with each other via nearestneighbor coupling which is called a spin-spin interaction. For this system, there is a competition between entropy that favors disorder in the spin orientations and spin alignment along one direction due to their spin-spin coupling energy, which is minimized for parallel alignment. At high temperatures, entropy "wins" and the system is disordered. At low temperatures, the opposite happens and the spins orient themselves along the same axis. The boundary between two ranges of temperature is called the critical point (or critical temperature) and close to a critical point very exotic behavior can be found where the system is infinitely sensitive to external stimuli such as magnetic fields and the dynamic behavior of the system's constituent spins is correlated over long distances such as small perturbations propagate from one end to the other. The two stable phases that arise are called a paramagnetic (disordered, also called symmetric) phase and a ferromagnetic (ordered, also called broken symmetry) state.

By analogy with the above example, we propose that linoleic acid's concentration in the membrane of neurons or glia acts as a control parameter (corresponding to temperature for magnetic systems), effectively behaving like a switch in a system close to criticality, i.e. resulting in a phase transition between a normal brain and a pathological brain when the linoleic acid concentration falls below its set value. It is important to emphasize that systems close to criticality exhibit long-range order which is important in this case so that an effect at a cell level can propagate macroscopically to the organ level, i.e. this is not just a local effect but a global effect that involves the entire brain. The question emerges, what would this change in concentration entail regarding the dynamics of the brain, in particular the functioning of a single neuron or astrocyte? Furthermore, it is important to understand the mechanics of such an effect exerting influence on the sub-neuronal constituents such as the cytoskeleton.

Recent observations, conducted on the characterization of the platelet fatty acids in mood disorders and ischemic heart disease have consistently shown that a very low concentration of linoleic acid is correlated with these diseases. This observation was found to be particularly interesting in brains of depressed subjects who had completed suicide. Moreover, it seem to be supported by the work of Lalovic et al. [9] for which, even if not significant, perhaps for a limited number of cases, it is noted that in the ventral prefrontal cortex (a decision making area in humans) of depressed or bipolar suicidal subjects there is a slight decrease of linoleic acid, as supported by a standard deviation of the data, compared to the control groups. Together with this observation, also the results achieved of a very low concentration of linoleic acid in platelets of subjects with suicide attempts, have encouraged researchers to consider whether, in fact, it was possible to record small and significant variations of linoleic acid in other circumstances. For this purpose, a research group from the School of Agriculture and Veterinary Medicine (University of Bologna) [data not published] has recently evaluated the fatty acid compositions of the brain of obese rats. In fact, in the brains of the obese rats, there was a statistically significant reduction of linoleic acid [10].

The set of variations in the concentration of linoleic acid in the brain, undoubtedly, becomes interesting from the perspective of interpretation regarding its position in the membrane and the likelihood that these minor changes can result in the connections that the membrane makes to the cytoskeleton through the interactions with the G protein, Gs $\alpha$ and ion channels. A mechanistic model of this interaction could be built starting with the fatty acids in the membrane forming an ordered network whose structure depends on the specific composition of the fatty acids, in particular the linoleic acid. To embark on developing such a model we first discuss how lessons from the physics of phase transitions can help us understand the possible consequences for neuroscience. 


\section{Predictions from the Ising model}

In fact, our proposal is an extension of an already existing extensive body of literature in membrane biophysics where the lipid composition of the membrane has been modeled in terms of the two-dimensional Ising model [11]. The Ising model is a standard approach to the modeling of critical phenomena in physics. This model predicts a transition between a disordered and an ordered phase of a material as a function of temperature or another control parameter which is coupled to the order parameter describing the gross features of the system. For magnetic systems, an order parameter is total magnetization and a control parameter is temperature. In the case of membrane implementations of the Ising model, the ordering structure involves the tails of the phospholipids composing the membrane so the order parameter could be the average membrane thickness for example while the control parameter could be the fatty acid composition of the membrane, for example the percentage amount of linoleic acid.

As mentioned above, a 2D Ising model is a classical example of a system exhibiting a phase transition between ordered and disordered state when the temperature is lowered below a critical point [12]. For the benefit of the reader, we explain the key features of the model in order to better understand how it can be made useful in applications to brain dynamics. The total energy of this type of magnetic system is calculated using the so-called Hamiltonian function, namely:

$$
H=-J \Sigma s_{i} s_{j}-B \Sigma s_{i}
$$

where $J$ is the interaction constant, $s_{i}$ is the spin, whose value can be either $-1 / 2$ or $1 / 2$, for the $i$ th magnetic particle in the spin lattice, $i$ and $j$ are indices labeling the adjacent particles and $B$ is the magnitude of the externally applied magnetic field that can be used to align spins along its direction irrespective of the temperature. In the absence of the external field $\mathrm{B}$, the transition to an ordered state occurs at a well-defined temperature given by the interaction strength constant $J$ :

$$
T_{c}=2.269 \mathrm{~J} / k_{B}
$$

This temperature, called the critical (or Curie) temperature has major significance because of the nonlinear behavior of the system at and near this value. The system exhibits extreme sensitivity to parameter changes when it approached the critical temperature from either above or below. Importantly, the nature of the spin ordering in the Ising model depends on spatial dimensionality (no transition above the absolute zero temperature occurs in the 1D case, for example), which is shown in Fig. 1. This figure demonstrates graphically the nature of ordering taking place in the system near criticality and some

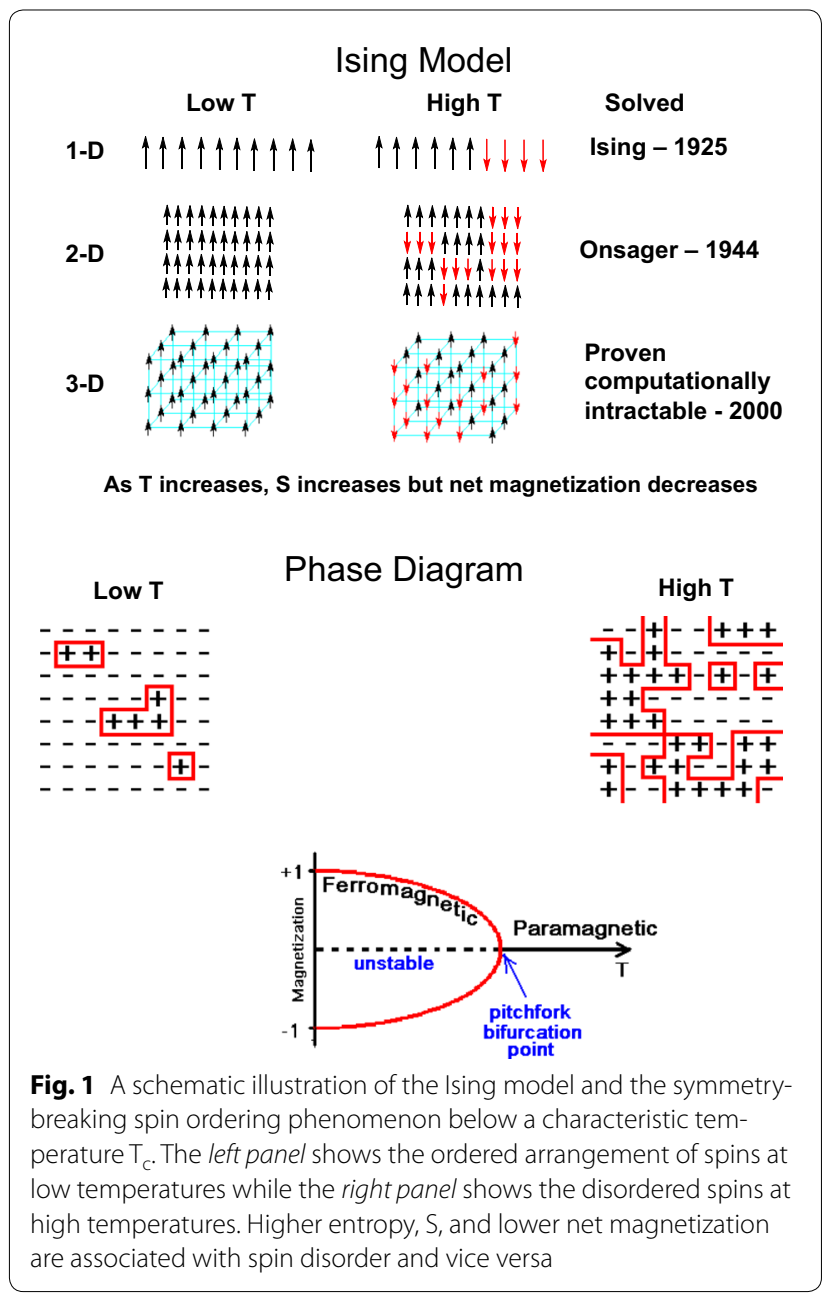

associated properties. This phenomenon exists in all dimensions except for linear systems (1D) but the values of the transition temperature change whether it is a $2 \mathrm{D}$ or a 3D system.

It is important to note that the Ising model is simply a blueprint for a physical effect in a system that can spontaneously reorganize itself when a specific parameter is varied. At criticality, the system is shown to be bistable, i.e. it can choose one of the two possible orientations for the ordering of the spin variables (all spins pointing up or all spins pointing down; with equal probability).

How can this be translated into the dynamics of membrane's phospholipids? Earlier work [11, 13] represented the orientation of the double bonds in fatty acid tails either as trans or cis, which can be mapped on the spin variable in the Ising model. In other words, we could consider a spin-up state as trans and a spin-down state as cis. Furthermore, instead of the temperature change causing an order-disorder transition in magnetic systems, one can introduce a parameter that defines a relative 
concentration of one type of fatty acid over another. In our case, the control parameter that drives the transition would be the linoleic acid concentration, whose critical value drives the system towards a phase transition causing a re-ordering of the double bonds in the membrane's fatty acids in the same manner as temperature approaching the critical value causes the spins in a magnet to correlate their orientations over large distances. In the case of a fatty acid organization of the membrane, this ordering of fatty acid tail states can additionally cause a mechanical rigidity change of the membrane as well as a major effect on the functioning of the ion channels due to the electro-mechanical membrane-protein coupling [14]. Below we discuss how this interaction can provide a link to quantum biology aspects of the functioning of neurons.

\section{The quantum Ising model and the interactions between membrane fatty acids and ion channels}

Interestingly, $1 \mathrm{D}$ and $2 \mathrm{D}$ Ising models have been also employed to describe the cooperative effects between ion channels in the membrane [15]. This means that ion channels operate synchronously and their activity can be controlled by the state of the membrane, in particular its rigidity and electrostatic trans-membrane potential that directly couples to the electric charge of the ions. This mechanism could bring a completely new perspective on the interpretation of quantum effects in the brain, which have been debated ever since the work of Penrose was published $[16,17]$. At the most fundamental level the argument for the need of quantum physical nature of the functioning of the brain has to do with non-algorithmic cognitive processes, which cannot be performed by classical computation. This led Penrose, Hameroff and others to speculate that the brain operates in the quantum regime $[18,19]$. How quantization of mental processes happens nobody really knows but it has been hypothesized that at least some brain dynamics occurs via delocalized quantum wave function operation [20]. This mechanism would confer clear advantages in the operation of cognitive functions in the brain and could explain some amazing feats of the human brain such as incredibly short reaction times of some athletes to situational changes or the ability of some geniuses to solve problems by intuitive insights without breaking these problems down into algorithmic subroutines. Instead, quantum search algorithms could be used that almost instantaneously explore the entire space of available states.

Interestingly, a quantum Ising model has been exactly solved mathematically and its solution leads to the existence of long-range correlations and wave function entanglement in a process that Penrose and his colleagues speculated about in connection with brain dynamics
[21]. However, in the present proposal, we postulate that the quantum coherence built by these interactions involves ions collectively passing through ion channels in the neuronal membrane, as a specific implementation of the quantum Ising model. As discussed above, the membrane's composition in terms of fatty acids can be viewed as acting as a control parameter, which could induce a classically ordered state (or not, depending on the linoleic acid's concentration). Hence the linoleic acid composition is as important as temperature in magnetic systems (obviously in human physiology temperature is kept fairly constant so it cannot play a critical role in the brain). This fatty acid organization, subsequently couples to the ion channels that can operate at a quantum level as we briefly discuss below. Note that in view of the above discussion, we predict that the fatty acid ordering phenomenon is described by a classical Ising model (where the variables have two real values: trans or cis, just like spins $+1 / 2$ or $-1 / 2$ ), while the ion channel dynamics is described using the quantum Ising model where the variables are quantum wavefunctions because ions can have a probabilistic distribution functions describing their location. The latter aspect has been recently elaborated on in mathematical detail by other authors [23, 24]. Figure 2 schematically illustrates how the tunneling of ions can form a basis for a quantum Ising model.

Furthermore, there may be a difference in hydrophobicity among different fatty acids of the brain and this may produce significant variations of the fatty acids dynamic of the phospholipid bilayer of neuronal membrane [22] This could affect the decoherence times of quantum excitations in the collective states of ions traversing the ion channels and in turn affect mental processes. Decoherence times define the duration of a quantum state before it manifests itself by a so-called wave function collapse meaning its actual value can be measured or observed. A quantum state can be a superposition state so that two or more specific states coexist. This can be metaphorically illustrated by having simultaneous thoughts of ordering sushi and pasta before making a decision. Once we order sushi, this is akin to the wave function collapse and making an actual choice. Decoherence time is the time it takes to collapse a quantum state. If it's too short, the system operates almost always classically. On the other hand, if the decoherence time is too long, quantum states could become too long-lived or trapped, and in the case of brain dynamics they may lead to obsessive thoughts and behaviors.

In a nutshell, what we suggest regarding the role of linoleic acid in this context is its effect on the ordered state of the cell membrane, which in turn affects the (possibly quantum) dynamics of the ion channels in neurons. Ion channels exhibit tunneling phenomena that have 


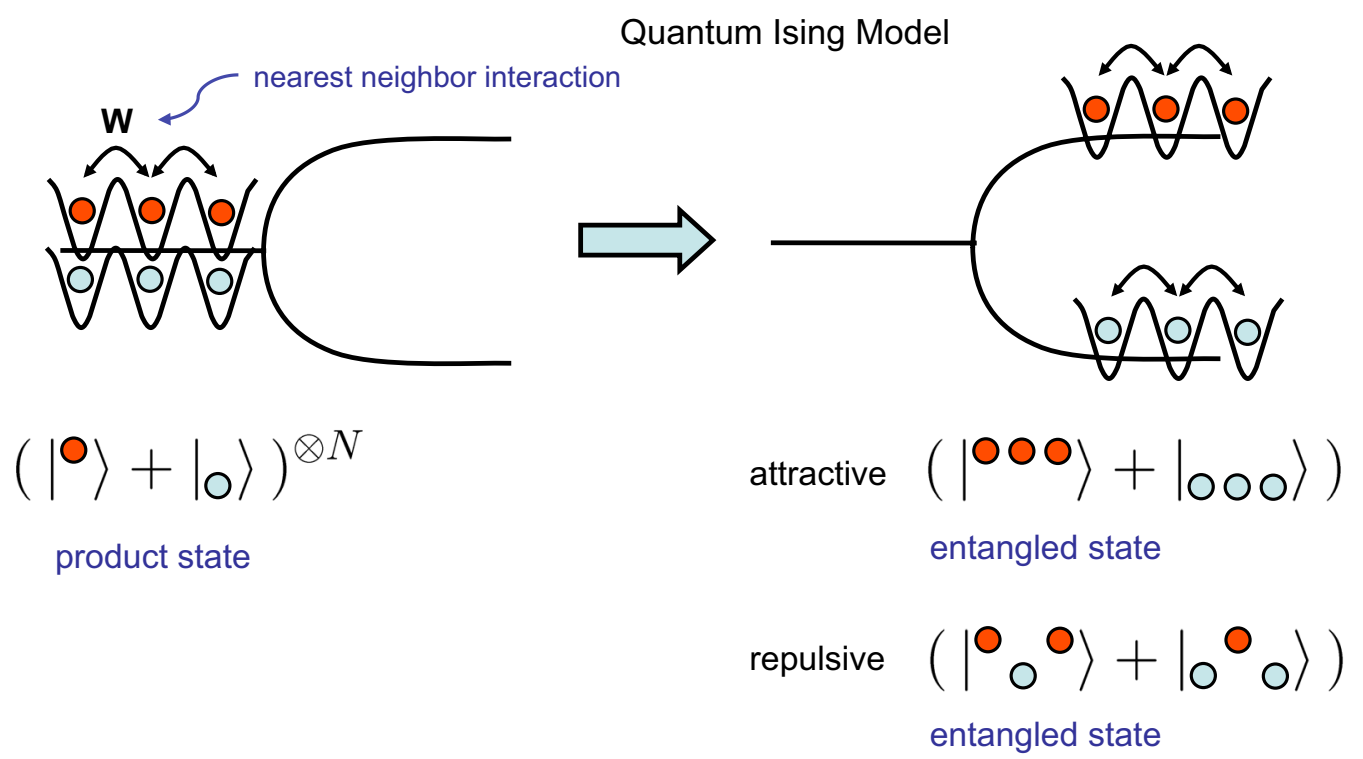

Fig. 2 lon tunneling in ion channels can be viewed as a basis for a quantum Ising model. A symmetric state on the left is a product state of ions occupying the two available quantum states marked red and blue. A broken symmetry state is shown on the right where the attractive and repulsive ionic arrangements are split into two distinct possibilities

been described by quantum mechanical Hamiltonians and hence offer a strong link between possible quantum effects in the membrane and in the cytoskeleton [23, 24]. There may be an additional connection to the functioning of the cytoskeleton as we describe below. This is very important in view of the involvement of the cytoskeleton in neuronal information processing as well as a host of neurodegenerative diseases [25].

\section{Zones of signal convergence: the interaction between ion channels and the cytoskeleton}

Electrical signaling in the brain builds on the concerted translocation of charges through integrated membrane channel proteins. Ever since the determination of the atomic resolution structure of channel proteins by MacKinnon et al. [26, 27], a highly detailed biophysical picture of the dynamics underlying the function of these proteins has been established. In particular, it was demonstrated that ion channels support quantum coherence resulting in characteristic resonances for the opening and closing of ion channel gates [23, 24].

Having outlined a possible connection between a detailed composition of the neuronal membrane and the functioning of ion channels, the next question to address is how this interaction can be further transmitted to the cytoplasm and the cytoskeleton. A connection to the cytoplasm can be readily provided by actin filaments that interact with the cell membrane [28]. There may also be a feedback mechanism operating in the opposite direction where the cytoskeleton affects the dynamics of the plasma membrane $[29,30]$. This latter effect is closely associated with $G$ proteins that act as shuttles between the membrane and both actin and tubulin filament networks [30].

The conventional mechanism of action potential involves the Hodgkin-Huxley model shown schematically in Fig. 3 and it just involves the membrane crossing ion fluxes propagating in and out of the cell via ion channels.

However, there is ample evidence that the cytoskeleton is involved in the action potential regulation, which would indicate a bidirectional communication between ion channels and the cytoskeleton as suggested above. Microtubule depolymerizing agents (e.g., colchicine) eliminate peripheral cytoskeleton and action potential together [31,32] and modify G protein signaling in mammalian cerebral cortex [33]. Microtubule stabilizing agents restore cytoskeleton and action potential together [31, 32]. Divalent ions such as magnesium and zinc restore cytoskeleton and action potential, according to Tasaki et al. [34]. On the other hand, calcium ions destabilize microtubules and actin filaments directly or indirectly by interactions with the various associated proteins. Consequently, the filaments of the cytoskeleton are likely to undergo highly localized reorganization in response to ion fluxes through the ion channels. Neurotransmitters, working through GPCRs can also alter microtubule dynamics, leading to changes in morphology 


\section{Conventional mechanism of action potential propagation:}
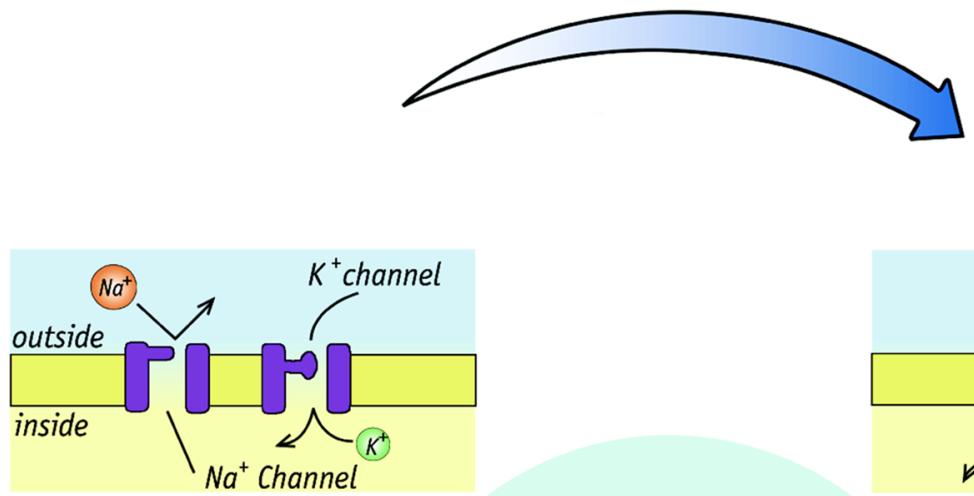

a resting state
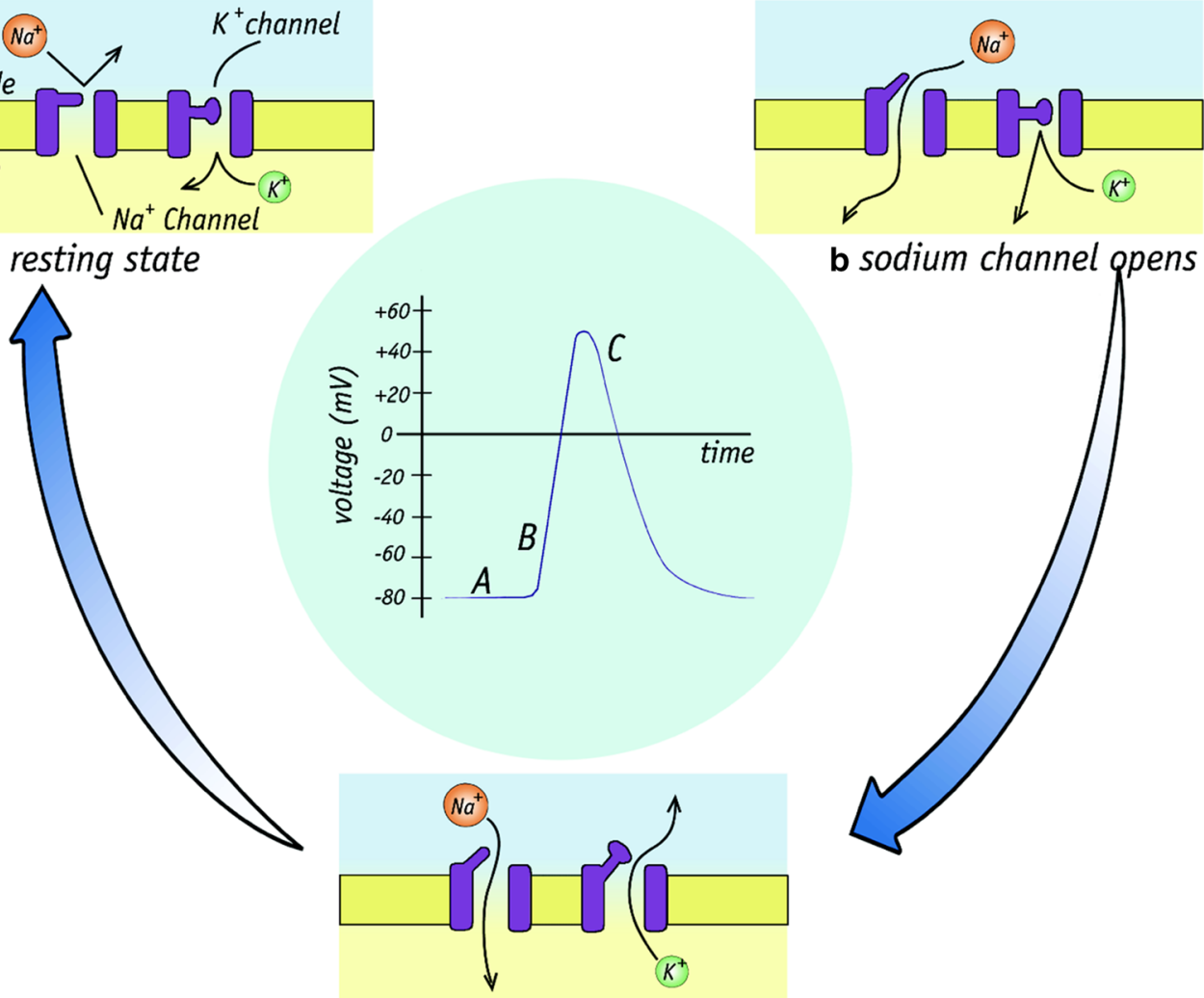

c potassium channel opens

Fig. 3 Schematic illustration of the standard representation of action potential propagation in neurons

of dendritic spines and the synapses located thereon [35, 36]. Conversely, microtubules and actin filaments may affect the opening and closing rates of ion channels. This has been extensively described in the work of G. Pollack [37] and is schematically illustrated in Fig. 4.

Following on Pollack's hypotheses, we believe that the cell's major communication systems (secretion, action potential generation) are governed by phase-transitions (symmetry breaking phenomena) taking place first in the structure of the membrane fatty acids, which are communicated to the negatively charged polymers of the cytoskeleton (actin filaments and microtubules) that are initially condensed by divalent cations. Then, monovalent cations $(\mathrm{Na}, \mathrm{K})$ trigger filament a decondensation transition due to ionic flows (see Fig. 5). This cytoskeletal transition is reversible as it depends on the ionic concentration. Note that the work of Rasenick et al. [35] suggests activated $G s \alpha$ is internalized through lipid rafts where it activates tubulin GTPase on the plus end of the microtubule, increasing microtubule dynamics and plasticity of synaptic structures such as spines.

It is interesting to consider the action of anesthetics in this connection. It is well known that they eliminate the propagation of action potentials. General anesthetics span a broad range of molecular shapes and sizes hence fail to fit into the "lock and key" paradigm. They exhibit no covalent chemistry but bind to hydrophobic regions of proteins and membranes. Anesthetics are also known to form water clathrates [38]. In a recent paper [39] anesthetics were shown to interact with numerous binding 


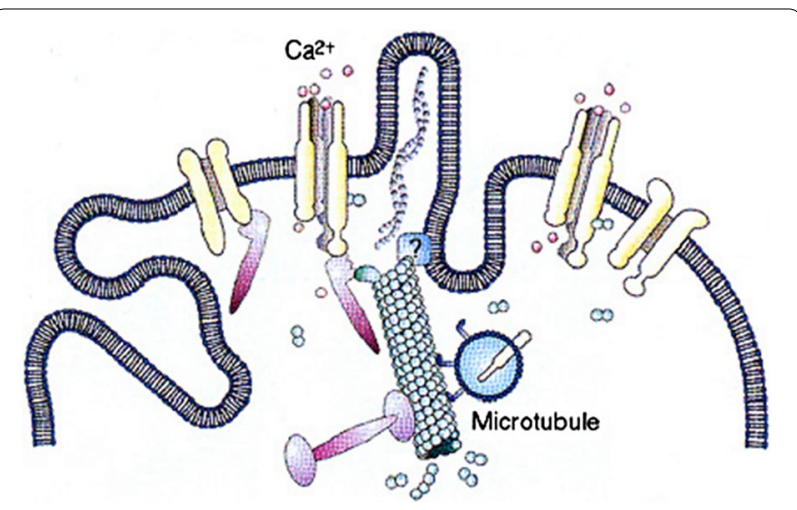

Fig. 4 A depiction of the microtubule interaction with calcium ion channels in the membrane and potentially directly with the membrane. A microtubule is shown depolymerizing at the minus end and providing a track for a kinesin molecule movement toward the plus end. The symmetric purple structure attached to the microtubule is a microtubule associate protein (MAP) while the asymmetric purple structures interacting with the ion channels and indirectly with the microtubule are KIF3B molecules

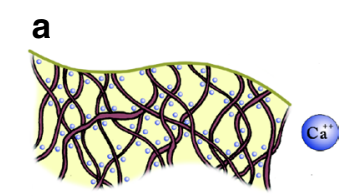

condensed state

b
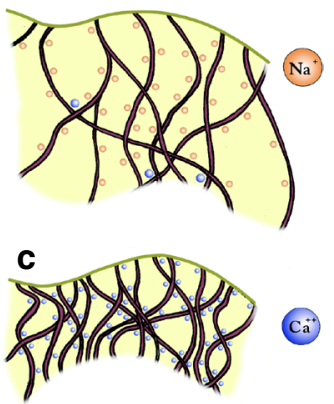

trigger

decondensation by sodium water entry--unzip increased permeability more sodium entry

water structure weakened retractive force collapses ntwk.

calcium rebinds

zips up network

sodium expelled

Fig. 5 Effects of calcium and sodium ion concentration changes affecting condensation and decondensation of cytoskeletal protein networks

sites on tubulin in addition to an earlier demonstration of binding to actin filaments. Consequently, anesthetics may exhibit multiple modes of action: direct binding to membranes that inhibits action potential propagation as well as binding to the cytoskeleton that may indirectly affect the cell's membrane. Moreover, according to Pollack, they could also inhibit action potential by preventing required water uptake into the cell [37].

One might also consider the relationship of antidepressants to the lipid microenvironment. Antidepressant compounds (save ketamine) require an extended period of treatment (up to 2 months) before they achieve clinical efficacy. Presumed targets of these drugs (monoamine transporters or catabolizing enzymes) are inhibited after a short treatment, so some other factor, perhaps an intrinsic property of the membrane, must also be implicated. Most known antidepressants have been shown to target lipid rafts [40] and this is a gradual process [41].

\section{The role of the cell membranes in psychopathology (neurons, glia and platelet)}

Plasma membrane of neurons and glia offer a promising way towards a better understanding of psychopathology in its different aspects: Major Depression, Bipolar Disorder and their effect on cognitive impairment. Several approaches have provided evidence about this subject. Rasenick et al. [42] have demonstrated that, in brain membranes from depressed suicides, that Gsa is enriched in lipid raft fractions where it is less complexed with adenylyl cyclase. The observation of diminished Gs adenylyl cyclase coupling was also been demonstrated in platelets $[43,44]$ and leukocytes. Thus, is suggested that the degree of lipid raft association of platelet Gs $\alpha$ might serve as a biological marker for depression.

Cocchi and Tonello [45-47] have studied the membranes of platelets of depressed subjects, highlighting the fatty acid profiles as a possible measure of the state of membrane.

The results obtained with the use of an artificial neural network (Self Organizing Map), have highlighted the possibility of classifying Mood Disorders by the so-called normality and, inside of Mood Disorders, to distinguish, with extreme precision, the subjects with Major Depression (MD) from those with Bipolar Disorder (BD). The results of the authors' experiments seem very consistent with those obtained on $G$ proteins associated with -plasma membrane lipid rafts delineated by the group of Rasenick et al. [42] as well as with the observations that the antibipolar drugs lithium and valproate, do not behave in a manner similar to antidepressants with regard to mobility of Gs $\alpha$ from lipid rafts [48]. The cell membrane lives, therefore, a constant condition of mobility, and, when the oscillations of fatty acids cross the tolerance ranges of the same, can be expressed pathological phenomena.

The mechanical factors of membrane lipids constitute lipid dependent factors that can dramatically affect protein functions or protein-protein interactions [49] and result in pathological consequences [22]. The condition of constant mobility of the membrane, whether for physiological levels or trespassing in pathological consequences, results in a deformation of the same at different levels of intensity with consequences that involve the raft lipid membrane, the protein $G s \alpha$, the cytoskeleton and the ion channels [50]. 
A number of factors influence the relationship between membrane and cytoskeleton, including the lipid composition, the density and the distribution of the cytoskeleton, the ratio between the surface area and volume, the internal cell pressure [51]. In the literature, the responses to the changes induced by forces of the mechanical type on the cytoskeleton have been extensively described [52-60].

All acquired knowledge, the study of quantum hypothesis of consciousness, the opportunity for dialogue that is open within the Quantum Paradigm Psychopathology Group (QPP), has certainly contributed to the construction of a new attempt hypothesis that seems to reconcile the previous ones, which, in reality, are only the result of different ways of looking at the same phenomenon.

The state of continual renewal and exchange of fatty acids by the membrane with consequent deformation of the lipid bilayer, can be thought of in terms of a mechanical force, in both physiological and pathological conditions, which is exercised on the cytoskeleton with continuity allowing, when there are physiological conditions for the membrane, periods of decoherence of microtubules (500 ms), which give rise to the conscious manifestation which will result in classic neurocorrelate of consciousness. The problem that arises is to understand how, under different conditions of mood disorders, the decoherence can be modified by lengthening or shortening the period $(<$ or $>500 \mathrm{~ms})$.
Having had the opportunity to understand that a higher mobility of membrane characterizes MDD and lower, BD, it is perceived that there are pathological conditions of deformation of the membrane that may exert different mechanical forces on the cytoskeleton from the inside of the same membrane and could potentially affect the physiological phenomenon of superposition of microtubules and consequently the period of oscillation.

In the case of BD there would be a shortening of the period of decoherence while in the case of MDD, an elongation. This eventuality could correspond, in cases of extreme criticality of decoherence period, to deep and decisive changes in the conscious state [22].

Having shown that between MDD and BD there is a symmetry break [61], keeping in mind the characteristics of mobility of the membrane, that there is a "domain wall" governed by electromagnetic forces that are likely to be the only form of communication between the two phenomena (MDD and BD) and that the only commonality of the two conditions is suicide, it seems consistent to think that such an event can take place for critical lengthening or shortening of the periods of decoherence, and, therefore, of the capacity of consciousness. These neuron forces, or "neuromechanics", could represent, the element conditioning the control domain of the microtubules and conditioning their relationship with the criticality of synapses, cortex and serotonin [62].

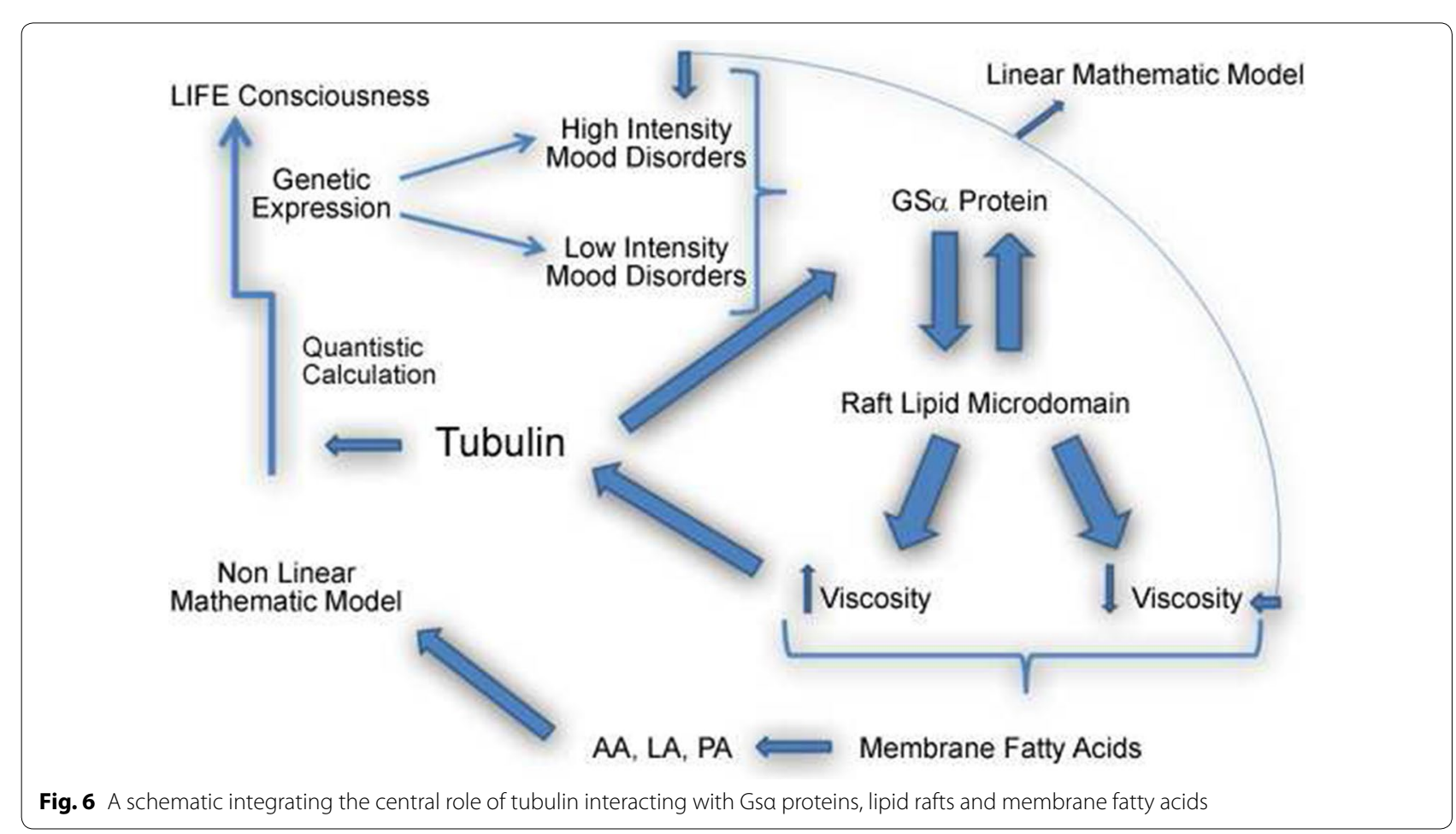




\section{Conclusions}

Much experimental evidence and consistent assumptions argue for a critical role of linoleic acid in the cell membranes. We know that is possible to measure very small changes of the brain Linoleic Acid's concentration in pathological conditions. What we cannot explain is what escapes to the phenomenology of events such as memory, consciousness, and even suicide. We are not able to understand the "sub microscopic level". We can measure the concentration of linoleic acid but we are not able to understand the complexity of the phenomena that it sets up as an end controller of the molecular fine-tuning, when, in the dynamics of the elements that relate to the cell between inside and outside, it affects the consciousness of individuals and affects their pathological responses.

The interactome, that is defined "as the whole [array of] molecular interactions that take place in an organism and allow the cascade of regulatory molecules including the mechanism of action of enzymes and metabolic reactions", it might respond to small, critical change in concentration of linoleic acid that are lost in the complexity of the quantum entanglement.

In this case, beyond the possible connections among serotonin, platelets, cytoskeletal proteins and other molecular interactions with implications regarding the genesis of the brain behavior. Cell membrane and fatty acids, in particular linoleic acid is the "key that opens to the quantum brain" through the dynamics of the cytoskeleton. Our proposed integrated picture of the classical/quantum dynamics at the level of a neuron but including the cytoskeleton is shown in Fig. 6.

The approach to the processes of mind-consciousness, in their normal and pathological dynamics, cannot, however, settle on isolated elements and plans, observable by a privileged and aloof observer, but thinking and acting operationally, by connections, relationships, networks. All this, always in the belief that science is narration of a world of expressive, emerging conditions-for this never reducible to the sum of its elementary ingredients-and in which the symmetry breaking provide variety, creativity, vitality, as a sign of self-natural organization, of evolving systems, thanks to spontaneous breakage of symmetry, towards conditions increasingly complex and unpredictable, including the person as an emerging relationship.

\section{Authors' contributions}

All authors contributed equally to the research presented in this article and to the writing of this manuscript. All authors read and approved the final manuscript.

\footnotetext{
Author details

1 "Paolo Sotgiu" Institute for Research in Quantitative \& Quantum Psychiatry \& Cardiology, L.U.de.S. HEI, Malta, Switzerland. ${ }^{2}$ Department of Veterinary Medical Sciences, University of Bologna, Bologna, Italy. ${ }^{3}$ UCC, University of Cork, Cork, Ireland. ${ }^{4}$ Neurosignaling Unit, Department of Organismic Biology,
}

University of Salzburg, Salzburg, Austria. ${ }^{5}$ Department of Oncology, University of Alberta, Edmonton, Canada. ${ }^{6}$ Department of Physics, University of Alberta, Edmonton, Canada. ${ }^{7}$ Department of Biomedicine and Neuroscience, University of Palermo, Palermo, Italy. ${ }^{8}$ Euro-Mediterranean Institute of Science and Technology, Palermo, Italy. ${ }^{9}$ Department of Physiology \& Biophysics and Psychiatry, University of Illinois College of Medicine, Chicago, IL, USA.

10 Jesse Brown VAMC, Chicago, IL, USA.

\section{Acknowledgements}

J.A.T. acknowledges funding from NSERC (Canada). M.M.R. is supported by the $\mathrm{NIH}$ (R01 AT009169) and the U.S. Veteran's Administration (BX001149).

\section{Competing interests}

The authors declare that they have no competing interests.

\section{Ethics approval and consent to participate}

Not applicable.

\section{Publisher's Note}

Springer Nature remains neutral with regard to jurisdictional claims in published maps and institutional affiliations.

Received: 19 January 2017 Accepted: 12 April 2017

Published online: 19 April 2017

\section{References}

1. Cocchi M, Tonello L, Gabrielli F, Minuto C. Human and animal brain phospholipids fatty acids, evolution and mood disorders. J Phylogenetics Evol Biol. 2014;2:1000128.

2. Svennerholm L. Distribution and fatty acid composition of phosphoglycerides in normal human brain. J Lipid Res. 1968;9:570-9.

3. Speake BK, Thompson MB, Thacker FE, Bedford GS. Distribution of lipids from the yolk to the tissues during development of the water python (Liasis fuscus). J Comp Physiol B. 2003;173:541-7.

4. Speake BK, Decrock F, Surai PF, Wood NA, Groscolas R. Establishment of the fatty acid profile of the brain of the king penguin (Aptenodytes patagonicus) at hatch: effects of a yolk that Is naturally rich in $\mathrm{n}-3$ polyunsaturates. Physiol Biochem Zool. 2003;76:187-95.

5. Cocchi M, Minuto C. Linoleic acid: a milestone in brain evolution? Hum Evol. 2015;30:245-57.

6. Simandle ET, Espinoza RE, Nussear KE, Tracy CR. Lizards, lipids, and dietary links to animal function. Physiol Biochem Zool. 2001;74:625-40.

7. Lawniczak CJ, Teece MA. Lipid metabolism during embryonic development of the common snapping turtle, Chelydra serpentina. Comp Biochem Physiol B Biochem Mol Biol. 2009;153:73-80.

8. Stanley HE. Introduction to phase transitions and critical phenomena. Oxford: Oxford University Press; 1987.

9. Lalovic A, Levy É, Canetti L, Sequeira A, Montoudis A, Turecki G. Fatty acid composition in postmortem brains of people who completed suicide. J Psychiatry Neurosci. 2007;32:363-70.

10. Cardenia V, Vivarelli F, Canistro D, Estrada MTR. Linoleic acid in brain rat and obesity disease: a new starting point for chronic disease? Commun Ital Soc Exp Biol (Sect Bologna). 2015 (Data not published).

11. Honerkamp-Smith AR, Veatch SL, Keller SL. An introduction to critical points for biophysicists; observations of compositional heterogeneity in lipid membranes. Biochim Biophys Acta. 2009;1788:53-63.

12. Yang CN. The spontaneous magnetization of a two-dimensional Ising model. Phys Rev. 1952;85:808-16.

13. Pink DA, Georgallas A, Zuckermann MJ. Phase transitions and critical indices of a phospholipid bilayer model. Z Physik B Condensed Matter. 1980;40:103-10.

14. Ashrafuzzaman M, Tuszynski JA. Membrane biophysics. Berlin: Springer; 2012.

15. Liu Y, Dilger JP. Application of the one- and two-dimensional Ising models to studies of cooperativity between ion channels. Biophys J. 1993:64:26-35.

16. Penrose R. Shadows of the mind. Oxford: Oxford University Press; 1994. 
17. Penrose R. The emperor's new mind: concerning computers, minds, and the laws of physics. Oxford: Oxford University Press; 1999.

18. Hameroff S, Penrose R. Orchestrated reduction of quantum coherence in brain microtubules: a model for consciousness. Math Comput Simul. 1996:40:453-80.

19. Hagan S, Hameroff SR, Tuszyński JA. Quantum computation in brain microtubules: decoherence and biological feasibility. Phys Rev E. 2002;65:61901.

20. Vattay G, Kauffman S, Niiranen S. Quantum biology on the edge of quantum chaos. PLoS ONE. 2014;9:e89017.

21. Cincio L, Dziarmaga J, Rams MM, Zurek WH. Entropy of entanglement and correlations induced by a quench: dynamics of a quantum phase transition in the quantum Ising model. Phys Rev A. 2007;75:52321.

22. Cocchi M, Tonello L, Gabrielli F. Possible roles of cell membrane and cytoskeleton in quantum aspect of psychiatry. J Conscious Explor Res. 2012:3:1082-1100.

23. Vaziri A, Plenio MB. Quantum coherence in ion channels: resonances, transport and verification. New J Phys. 2010;12:85001.

24. Summhammer J, Salari V, Bernroider G. A quantum-mechanical description of ion motion within the confining potentials of voltage-gated ion channels. J Integr Neurosci. 2012;11:123-35.

25. Woolf NJ, Priel A. Nanoneuroscience: structural and functional roles of the neuronal cytoskeleton in health and disease. Berlin: Springer; 2009.

26. Doyle DA, Morais Cabral J, Pfuetzner RA, Kuo A, Gulbis JM, Cohen SL, et al. The structure of the potassium channel: molecular basis of $\mathrm{K}+$ conduction and selectivity. Science. 1998;280:69-77.

27. MacKinnon R. Potassium channels. FASEB Lett. 2003;555(1):62-5.

28. Machta BB, Papanikolaou S, Sethna JP, Veatch SL. Minimal model of plasma membrane heterogeneity requires coupling cortical actin to criticality. Biophys J. 2011;100:1668-77.

29. Ehrig J, Petrov EP, Schwille P. Near-critical fluctuations and cytoskeletonassisted phase separation lead to subdiffusion in cell membranes. Biophys J. 2011;100:80-9.

30. Schappi JM, Krbanjevic A, Rasenick MM. Tubulin, actin and heterotrimeric $G$ proteins: coordination of signaling and structure. Biochim Biophys Acta. 2014;1838:674-81.

31. Matsumoto G, Ichikawa M, Tasaki A. Axonal microtubules necessary for generation of sodium current in squid giant axons: II. Effect of colchicine upon asymmetrical displacement current. J Membrain Biol. 1983;77:93-9.

32. Matsumoto G, Ichikawa M, Tasaki A, Murofushi H, Sakai H. Axonal microtubules necessary for generation of sodium current in squid giant axons: I. Pharmacological study on sodium current and restoration of sodium current by microtubule proteins and $260 \mathrm{~K}$ protein. J Membrain Biol. 1983;77:77-91.

33. Rasenick MM, Stein PJ, Bitensky MW. The regulatory subunit of adenylate cyclase interacts with cytoskeletal components. Nature. 1981;294:560-2.

34. Tasaki I, Singer I, Takenaka T. Effects of internal and external ionic environment on excitability of squid giant axon. J Gen Physiol. 1965;48:1095-123.

35. Sarma T, Koutsouris A, Yu JZ, Krbanjevic A, Hope TJ, Rasenick MM. Activation of microtubule dynamics increases neuronal growth via the nerve growth factor (NGF)- and Gas-mediated signaling pathways. J Biol Chem. 2015;290:10045-56

36. Popova JS, Rasenick MM. G beta gamma mediates the interplay between tubulin dimers and microtubules in the modulation of $\mathrm{Gq}$ signaling. J Biol Chem. 2003;278:34299-308

37. Pollack GH. Cells, gels and the engines of life: a new, unifying approach to cell function. Seattle: Ebner \& Sons; 2001

38. Pauling L. A molecular theory of general anesthesia: anesthesia is attributed to the formation in the brain of minute hydrate crystals of the clathrate type. Science. 1961;134:15-21.

39. CraddockTJA, George MS, Freedman H, Barakat KH, Damaraju S, Hameroff $\mathrm{S}$, et al. Computational predictions of volatile anesthetic interactions with the microtubule cytoskeleton: implications for side effects of general anesthesia. PLoS ONE. 2012;7:e37251.

40. Eisensamer B, Uhr M, Meyr S, Gimpl G, Deiml T, Rammes G, Lambert JJ, Zieglgänsberger W, Holsboer F, Rupprecht R. Antidepressants and antipsychotic drugs colocalize with 5-HT3 receptors in raft-like domains. J Neurosci. 2005;25:10198-206.

41. Erb SJ, Schappi JM, Rasenick MM. Antidepressants accumulate in lipid rafts independent of monoamine transporters to modulate redistribution of the G protein, Gas. J Biol Chem. 2016;291:19725-33.

42. Donati RJ, Dwivedi Y, Roberts RC, Conley RR, Pandey GN, Rasenick MM. Postmortem brain tissue of depressed suicides reveals increased Gsa localization in lipid raft domains where it is less likely to activate adenylyl cyclase. J Neurosci. 2008;28:3042-50.

43. Hines LM, Tabakoff B. Platelet adenylyl cyclase activity: a biological marker for major depression and recent drug use. Biol Psychiatry. 2005;58:955-62.

44. Mooney JJ, Samson JA, McHale NL, Pappalarado KM, Alpert JE, Schildkraut JJ. Increased Gsa within blood cells membrane lipid microdomains in some depressive disorders: an Exploratory study. J Psychiatry Res. 2013:47:706-11.

45. Cocchi M, Tonello L. Bio molecular considerations in major depression and ischemic cardiovascular disease. Cent Nerv Syst Agents Med Chem. 2010;10:97-107.

46. Cocchi M, Tonello L, Rasenick MM. Human depression: a new approach in quantitative psychiatry. Ann Gen Psychiatry. 2010;9:25.

47. Tonello L, Cocchi M. The cell membrane: is it a bridge from psychiatry to quantum consciousness? NeuroQuantology. 2010;8:54-60.

48. Donati RJ, Schappi J, Czysz AH, Jackson A, Rasenick MM. Differential effects of antidepressants escitalopram versus lithium on Gs alpha membrane relocalization. BMC Neurosci. 2015;16:40.

49. Allen JA, Halverson-Tamboli RA, Rasenick MM. Lipid raft microdomains and neurotransmitter signalling. Nat Rev Neurosci. 2007;8:128-40.

50. Cocchi M, Gabrielli F, Tonello L, Pregnolato M. The interactome hypothesis of depression. NeuroQuantology. 2010;4:603-13.

51. Mclntosh TJ, Simon SA. Roles of bilayer material properties in function and distribution of membrane proteins. Annu Rev Biophys Biomol Struct. 2006:35:177-98.

52. Chifflet $\mathrm{S}$, Hernández JA. The plasma membrane potential and the organization of the actin cytoskeleton of epithelial cells. Int J Cell Biol. 2012;2012:121424.

53. Sun M, Northup N, Marga F, Huber T, Byfield FJ, Levitan I, et al. The effect of cellular cholesterol on membrane-cytoskeleton adhesion. J Cell Sci. 2007;120:2223-31.

54. Yap B, Kamm RD. Cytoskeletal remodeling and cellular activation during deformation of neutrophils into narrow channels. J Appl Physiol. 2005;99:2323-30.

55. Hoover RL, Fujiwara K, Klausner RD, Bhalla DK, Tucker R, Karnovsky MJ. Effects of free fatty acids on the organization of cytoskeletal elements in lymphocytes. Mol Cell Biol. 1981;1:939-48.

56. Apodaca G. Modulation of membrane traffic by mechanical stimuli. Am J Physiol Renal Physiol. 2002;282:F179-90.

57. Namikoshi M, Suzuki S, Aaronson S, Kobayashi H, Mine Y, Kasuga I. Inhibitors of microtubule assembly produced by the marine fungus strain TUF 98F139 collected in Palau. J Tokyo Univ Fish. 2002:88:1-6.

58. Janmey P. Cell membranes and the cytoskeleton. Handb Biol Phys. 1995;1:805-49.

59. Janmey PA, McCulloch CA. Cell mechanics: integrating cell responses to mechanical stimuli. Annu Rev Biomed Eng. 2007;9:1-34.

60. Chalfie M. Neurosensory mechanotransduction. Nat Rev Mol Cell Biol. 2009;10:44-52.

61. Cocchi M, Gabrielli F, Pessa E, Pregnolato M, Tonello L, Zizzi P. Major depression and bipolar disorder: the concept of symmetry breaking. NeuroQuantology. 2010;10:676-87.

62. Cocchi M, Tonello L, Gabrielli F, Pregnolato M, Pessa E. Quantum human \& animal consciousness: a concept embracing philosophy, quantitative molecular biology and mathematics. J Conscious Explor Res. 2011;2:547-74 